\title{
OXIDATIVE STRESS AND CARDIOVASCULAR DISEASES
}

\author{
M. Hristova*, M. Penev \\ Department of Internal Medicine, Medical Faculty, Trakia University, Stara Zagora, Bulgaria
}

\begin{abstract}
Reactive oxygen species (ROS) are reactive oxygen metabolites resulting from one-electron reduction of oxygen under the influence of a variety of enzymes. In aerobic organisms, they are produced as a byproduct of many cellular metabolic pathways and participate in some processes necessary for the normal life and functioning of the cells, but also in a large number of pathological processes.

Oxidative stress is a molecular dysregulation of ROS metabolism, which plays a leading role in the pathogenesis of atherosclerosis, vascular inflammation and endothelial dysfunction. The good understanding of the role of oxidative stress in vascular pathology aims to create new therapeutic strategies for stabilization of the endothelial function by reduction of oxidative stress.
\end{abstract}

Key words: reactive oxygen species, oxidative stress, endothelial dysfunction, atherosclerosis

Generation of ROS in living organisms. Pathology of their overproduction for the individual.

The paradox of aerobic life is that the life-giving oxygen is lethally toxic as well! Free radicals are the price aerobic organisms pay for the use of oxygen! (1)

In biological systems the formation of free radicals occurs mainly based on the following chemical processes (2):

1. Homolytic cleavage of covalent chemical bonds (most often).

2. Single-electron redox processes.

3. Singlet-triplet transitions (for electron excitation of molecules).

4. Exchange chemical reactions to form radical intermediates.

According to their reactivity radicals are classified as relatively low reactive and highly reactive (2). ROS are reactive oxygen metabolites resulting from one-electron reduction of oxygen under the influence of a variety of enzymes (2). In living organisms they are produced as a by-product from a number of

*Correspondence to: Mariyana Kostova Hristova, 2 Ivan Kirov Vazov Street Apt.14, Zheleznik Quarter, 6014 Stara Zagora, Bulgaria, Phone: 0896329990 , Email address: MC_girl.87@abv.bg cellular metabolic pathways and influence normal and abnormal cell processes, including cell growth and hypertrophy, inflammation, remodelling, oxidation of lipids and vascular tone modulation. These include superoxide anion radical $\mathrm{O}_{2}{ }^{-}$, hydrogen peroxide $\mathrm{H}_{2} \mathrm{O}_{2}$, hydroxyl radical $\mathrm{OH}$, hypochlorous acid HOCL, reactive aldehydes, lipid peroxides $\mathrm{ROOH}$, lipid radicals and NO. Some ROS are radicals with unpaired valent electrons $\left(\mathrm{O}_{2}{ }^{-}, \mathrm{OH} \cdot \mathrm{NO}\right)$, others $\left(\mathrm{H}_{2} \mathrm{O}_{2}\right.$, HOCL) are biologically active oxidants, but are not radicals (3).

In mammalian cells, potential enzymatic sources of ROS include mitochondrial electron transport, lipoxygenase and cyclooxygenase enzymes of the arachidonic cascade, cytochrome p450 enzymes, xanthine oxidase, NADH/NADPH oxidase, nitric oxide synthetase (NOS), peroxidases and other hemoproteins (4). Although many of these sources can produce ROS and inactivate NO, three have been extensively studied for the cardiovascular system: 1 . xanthine oxidase 2. NADH/NADPH 3. NOS

An emphasis is placed on the vascular membrane associated NADH/NADPH oxidase, expressed on endothelial cells, vascular cells and fibroblasts comprising the main source of ROS in blood vessels (5). 


\section{Oxidative stress}

According to Schramm et al., oxidative stress represents molecular dysregulation of the ROSmetabolism, which plays a key role in the pathogenesis of atherosclerosis, vascular inflammation and endothelial dysfunction and is characterized by loss of NO-bioavailability (6). The large clinical trials such as HOPE (2000) and HPS (2002) showed no clinical benefit (7) from the use of antioxidant vitamin $\mathrm{C}$ or vitamin $\mathrm{E}$ treatment, questioning the role of oxidative stress in cardiovascular diseases. The change in the understanding of the molecular nature of oxidative stress is due to the results of these studies (6). According to Schramm et al. (6) oxidative stress is no longer seen as a simple imbalance between the production and elimination of ROS, but also as a dysfunction of enzymes involved in the production of ROS. NADPH oxidase is given a key role, placed in the basis of the dysfunction of other oxidases, including "uncoupling" of the endothelial NOS (eNOS), xanthine oxidase and mitochondrial dysfunction (6). Therefore, oxidative stress is a term used to explain the conditions in which there is imbalance between the cellular levels of oxidants and antioxidants (3), wherein the electron transfer, cell function and enzymatic function are impaired. Förstermann U determined oxidative stress as primarily caused by an imbalance between the activity of the endogenous pro-oxidative enzymes (such as NADPH oxidase, xanthine oxidase, or mitochondrial respiratory chain) and antioxidant enzymes (superoxide dismutase (SOD), glutathione peroxidase, heme oxygenase, thioredoxin peroxidase (peroxiredoxin), catalase, paraoxonase) in favour of the former (8).

\section{Physiological relevance of ROS. Conditions associated with dysregulation of the ROS- metabolism.}

Harrison et al. considering the cytotoxic role of ROS, also highlight their key role in the normal existence and functioning of cells (3). Superoxide and $\mathrm{H}_{2} \mathrm{O}_{2}$ can act as signalling molecules to activate gene transcription (through transcription factors activator protein 1 (Ap-1), nuclear factor $\mathrm{kB}(\mathrm{NF}-\mathrm{kB})(9,10)$, etc. $)$, participate in the regulation of cell growth, endothelial function, inflammatory response, deposition of extracellular matrix (3). Touyz emphasized that molecular processes related to ROS, induced vascular changes including activation through activation of redox-sensitive signalling pathways (cascade processes) (10).

According to the latter, superoxide and $\mathrm{H}_{2} \mathrm{O}_{2}$ stimulate mitogen-activated protein kinases (which in turn regulate the transcription), tyrosine kinases, inactivate protein tyrosine phosphatase. $(10,11,12)$

$\mathrm{H}_{2} \mathrm{O}_{2}$ can also act as a direct vasodilator in some vascular beds, including the cerebral, coronary and mesenteric arteries $(13 ; 14-16)$, while the increased vascular levels of $\mathrm{O}_{2}{ }^{-}$inactivate the vasodilator NO and, respectively, lead to dysfunction and vasoconstriction. $(13,17,18)$

Reactive oxygene metabolites play a prominent role in $\mathrm{Ca}^{2+}$ homeostasis (19). In recent years, $\mathrm{Ca}^{2+}$ homeostasis is the subject of increasing interest, given the fact that disorders concerning calcium transport can seriously affect cellular functions. (Figure 1)

ROS activate protooncogenes and proinflammatory gene expression $(10,11)$. These processes are carried out by the oxidative modification of proteins, alteration of essential amino acid residues, induction of proteindimerization, reaction with metal complexes, such as $\mathrm{Fe}-\mathrm{S}$ residues. Changes in the intracellular redox state by the thioredoxin and glutathione systems can affect signalling pathways (10-12).

\section{Synthesis of NO- conditions and function}

The main reactive nitrogen species (RNS) is NOpotent vasodilator responsible for the endothelium-dependent vasorelaxation. Nitric oxide also has anti-inflammatory and antimitogenic properties and because of its uniqueness it was nominated molecule of the year in $1992 .(13 ; 21)$

NO is the smallest known signal molecule produced from three isoforms of the NOS enzyme. They all use L-arginine and molecular oxygen as substrates and as cofactors they require reduced nicotinamide adenine dinucleotide phosphate (NADPH), flavin adenine dinucleotide (FAD), flavin mononucleotide (FMN) and 5,6,7,8tetrahydrobiopterin (BH(4)). All NOS bind calmodulin and contain haem. Neuronal NOS (n NOS, NOS I) is expressed in the central and peripheral neurons and other cell types. The function of the enzyme includes synaptic plasticity in the CNS, central blood pressure 
regulation, smooth muscle relaxation and vasodilation via peripheral nerves (important for the relaxation of the corpus cavernosum and the erection of the penis). Inducible NOS (NOS II) can be expressed in many cell types in response to lipopolysaccharides, cytokines and other agents. Inducible NOS generates large quantities of NO, which have cytostatic effect on target "parasitic cells". NOS II contributes to the pathophysiology of inflammatory diseases and septic shock.

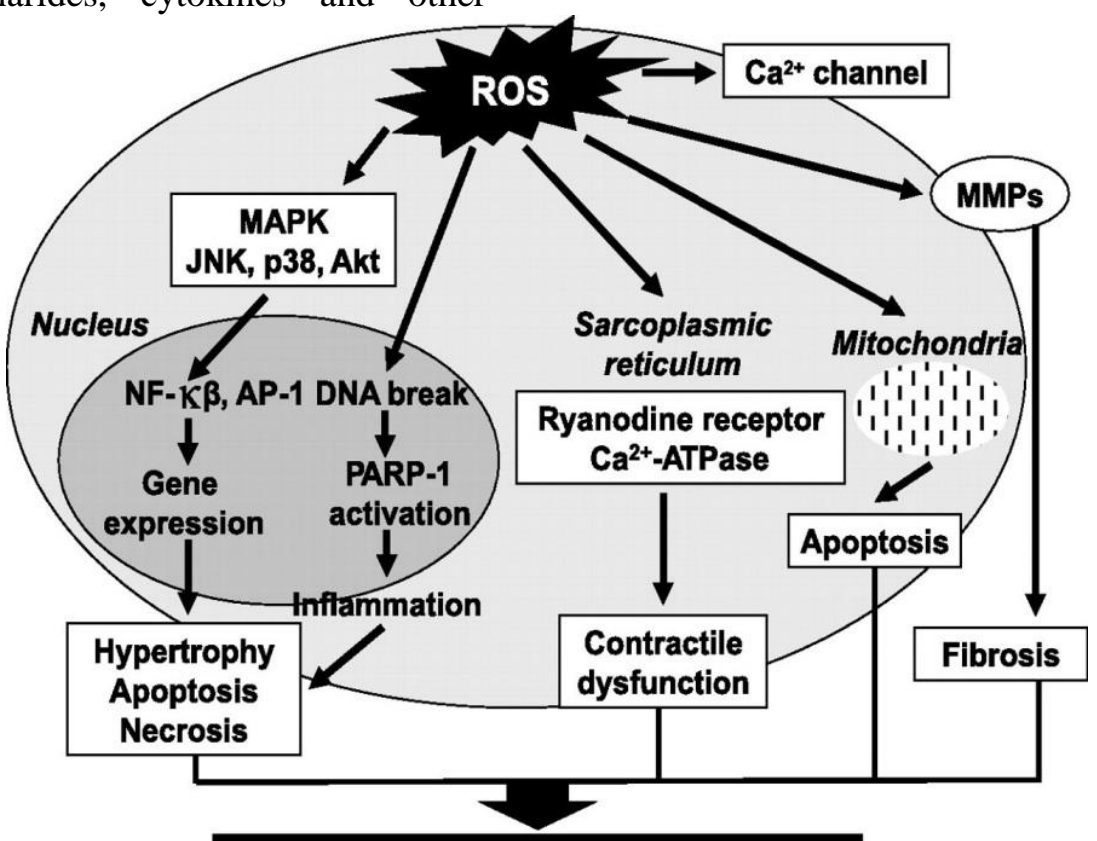

\section{Cardiac remodeling and fallure}

Figure 1. Cardiac remodeling and failure (by Tsutsui, Kinugawa, Matsushima 2011 (20)

Endothelial NOS (NOS III, eNOS) is expressed predominantly in endothelial cells. It contributes to the vasodilatation, control of arterial blood pressure (ABP) and has a number of antiatherosclerotic effects.

Many cardiovascular risk factors lead to oxidative stress, eNOS, "uncoupling" and endothelial dysfunction in vessels (22). VasquezVivar, Andrew et al. believe that the three NOS isoforms are able to "disengage" (uncoupling), potentially leading to the formation of $\mathrm{O}_{2}^{-}(13$, $23,24)$.

The three isoforms of NOS are named based on the tissue in which they were first described. Sugamura and John, citing the works of Griffith OW et al., believe that in most cardiovascular tissues $\mathrm{n}$ NOS and e NOS are expressed and present constitutively, but they have different subcellular localisation. In contrast, NOS II is usually missing and it is induced by proinflammatory mediators $(25,26)$.

Nitric oxide can be generated by other redox enzymes, including xanthine oxidase $(13,27)$.

\section{Endothelial dysfunction}

The term endothelial dysfunction (ED) is used to refer to several pathological conditions, including altered anticoagulant and antiinflammatory properties of the endothelium, impaired modulation of vascular growth and dysregulated vascular remodelling (4). However, Cai and Harrison (4) report that in great part of the literature this term is used to refer to damage to the endothelium-dependent vasodilation caused by loss of NO-bioactivity in the vessel wall. The latter (4), referring to the results of some studies on humans have claimed that traditional risk factors for atherosclerosis predispose to ED. Impaired endotheliumdependent vasodilation in the coronary circulation in humans predicts adverse cardiovascular events in the long term (4). According Madau et al. (28) the pathogenesis of ED is multi-factorial, but oxidative stress is placed at the base of the cell mechanism with a subsequent loss of the vaso-active substances, inflammation, disorder in homeostasis and redox homeostasis in the vascular system of the body. These authors (28) considered the role of ED as a pathophysiological link between early endothelial cell changes associated with 
cardiovascular risk factors and the development of ischemic heart disease (IHD) and arterial hypertension $(\mathrm{AH})$, which are essential for the clinician. Persistent high levels of circulating noxious stimuli associated with cardiovascular risk factors, cause sequential reactions occurring in endothelial cells - endothelial cell activation and ED, characterized by reduced nitric oxide concentration - an early reversible precursor of atherosclerosis (28).

The reduced NO bioavailability could be due to reduced expression of e NOS $(4,29)$, absence of a substrate or cofactors for e $\operatorname{NOS}(4,30)$, a change in cellular signalling, leading to abnormal activation of e NOS (4, 31), accelerated degradation of NO mediated by ROS $(4,32)$.

Earlier studies $(4,33)$ show that the endothelial derived relaxing factor (EDRF) identified as NO was inactivated by $\mathrm{O}_{2}^{-}$and stabilized by the superoxide dismutase (SOD). It should be borne in mind that the reaction between $\mathrm{O}_{2}{ }^{-}$and $\mathrm{NO}$ proceeds at a rapid rate - three times faster than the rate of the reaction between $\mathrm{O}_{2}{ }^{-}$and SOD (4, 34). Under normal physiological conditions, dynamic balance is maintained between the $\mathrm{O}_{2}$ and NO. This poor balance is altered in various disease conditions. For example, it has been found experimentally in hypercholesterolemic rabbits that NO- bioavailability is reduced by $\mathrm{O}_{2}$ (4, 35). Similar observations associated with increased degradation of NO by ROS in animal models include $\mathrm{AH}, \mathrm{DM}$, smoking, heart failure $(4,36-39)$. Studies continue in humans.

Considering the functional and structural vascular abnormalities in patients with $\mathrm{AH}$, Sudano et al. found that in addition to the reduction of $\mathrm{NO}$, endothelial dysfunction is associated with increased arterial stiffness (40). This parameter is considered as a sign of cardiovascular risk and its measurement in patients with $\mathrm{AH}$ is recommended by the European guidelines for the diagnosis and treatment of hypertension (40).

Mervaala, E. et al. found that endothelial dysfunction in transgenic rats with superexpression of renin and angiotensinogen is associated with increased activity of XO (13, 41).

\section{Cardiovascular risk factors and endothelial dysfunction}

To conventional risk factors (based on a sample of 2883 participants in the Framingham study) Halcox and Quyyumi consider new ones, including hyperhomocysteinemia, a sedentary lifestyle, exposure to pathogens - Chlamydia pneumoniae, cytomegalovirus (CMV), herpes simplex, Helicobacter pylori and hepatitis viruses (42). In search of new therapeutic approaches to reduce atherosclerotic and inflammatory vascular pathology, Charakida et al. found that patients with chronic inflammatory diseases are also at increased cardiovascular risk (43), and as one of the factors for atherosclerotic progression in them the authors suggest increased oxidative stress (43). The new procedures considered by the authors (43) are directed to inflammatory pathways and have beneficial effects on rheumatic diseases; however, their impact on reducing the cardiovascular risk remains unclear (43). Among the main causes of chronic inflammation accompanying atherogenesis, Yin et al. indicate IL1 and IL 18 (44). Their synthesis is mediated by ROS, facilitating the assembly of multiprotein complexes - inflammasomes, which activate caspase 1 .

According Halcox and Quyyumi the interaction of the individual genotype and the vascular phenotype should not be disregarded (42). The authors refer to the fact that the polymorphism of several genes contributes to the modulation of expression of proteins in various molecular pathways that can alter the endothelial function, respectively, the risk of atherosclerosis (42).

\section{ROS and atherosclerosis}

According to Kuyumdzhieva and Petrova there is sufficient evidence for the role of free radicals in just two disorders: oxygen deficiency and $\mathrm{CCl}_{4}$ - induced liver damage. Other diseases are in a process of theoretical and experimental work on the key role of ROS as a cause or consequence of the disease (19).

Atherosclerosis is a chronic inflammatory disease associated with certain risk factors (hypertension, DM, hyperlipidemia), characterized by hardening and thinning of the arterial wall (1). There are numerous ways by which increased ROS production participates in the development of atherosclerotic lesions. For an explanation of the pathogenesis of atherosclerosis Parthasarathy proposes the 
oxidative modification theory. According to it the free-radical processes and lipid peroxidation are decisive for the initiation of atherogenesis and atherosclerotic plaque formation (2).

Under normal circumstances, the native LDL circulates inside and outside the vascular wall. Than could be changed under certain conditions. Changes in the oxidative environment of the vascular wall favour the LDL oxidation and promote an inflammatory response, in part, through effect on Toll-like (TLRs) receptors. (3) Yin et al. consider the important pathogenic role of TLRs in the atherosclerotic process, emphasising that genetic deficiency of TLRs (TLR2; TLR 4) in atherogenic mice significantly reduces the size of atherosclerotic lesions (44). Since plasma inhibits LDL oxidation, it appears that, for the most part, the oxidation of the polyunsaturated fatty acids in LDL takes place inside the arterial walls (19). The substantial oxidation of LDL results in the formation of oxLDL is no longer recognised by the LDL receptor but is readily taken up by scavenger receptors. Oxidation of LDL results in the formation of several biologically active molecules. For example, ox-LDL contains phospholipase A2-like enzyme activity in LDL particles. The active conversion of phosphatidylcholine to lysophosphatidylcholine causes many adverse biological effects on the endothelium. Linolenic acid and other fatty acids are oxidized to the corresponding hydroperoxides participating in radical chain reactions which lead to the transfer of electrons to other molecules and formation of additional radicals (3). Once oxidized in the tissues, macrophages and monocytes can phagocytose the modified LDL, which results in foam cells, necessary for the formation of "fatty deposits" (precursor state of atherosclerosis) (19).

According to Tsvetkov and Bochev (2) initial oxidation of LDL activates the expression of genes which induces the production of monocyte activators - P-selectin, monocyte colony stimulating factor MCSF, VCAM1, X-LAM adhesion molecule, monocyte chemoattractant protein MCP-1 (2). The oxidized LDL stimulates the expression of $\mathrm{Mn}-\mathrm{SOD}$, which in turn increases the concentration of $\mathrm{H}_{2} \mathrm{O}_{2}(1)$.

As a critical component of vascular growth and remodelling Harrison and Griendling (3) examine the degradation and re-synthesis of the extracellular matrix. The process is mediated by specialised enzymes called matrix metalloproteinases (MMPs). Several MMPs, including MMP2 and MMP9, involved in degradation of the basement membrane and elastin, are converted from inactive zymogens into their active form by the ROS-mediated oxidation at the cysteine residue in the inhibitory domain. That process appears to cause matrix degradation in the areas of the vessels exposed to elevated levels of oxidative stress, such as the area of the "elbow" of vulnerable plaque (3).

Tsvetkov and Bochev identified as a triggering factor for atherogenesis the endothelial cell lesion (2). As one of the leading causes of endothelial damage authors emphasise the reactive metabolites of oxygen and the products of lipid peroxidation (2). On the other hand, the damaged endothelium is reacted with increased radical formation on the site of the vascular lesion. In support of the latter statement, Juni et al. examined the increased formation of ROS by various molecular mechanisms following coronary intervention, balloon angioplasty, stent implantation -BMS or DES (45). Thus a vicious circle increasing the vascular lesion is closed.

Inflammation plays an important role in all stages of atherosclerosis.

Stable plaques are characterized by a chronic inflammatory infiltrate and vulnerable and ruptured plaques are characterized by active inflammation participating in thinning of the fibrous cap and predisposing to plaque rupture (46). There is much evidence in recent years indicating that ROS are an important component of the inflammatory process. $(19,46)$ Regardless of their beneficial effect in destroying the invading microorganism and the generation of hematologic factors, the latter can result in tissue damage as well. The majority of ROS, produced as part of the inflammatory response, clearly come from various phagocytic cells, which when activated, are capable of producing a large quantity of superoxide anions and hydrogen peroxide. Those ROS can be coupled with free iron (which may be incurred by ROS-mediated release of chelated iron) to form highly reactive hydroxyl radicals. The latter cause significant tissue damage, which is not caused by the initial inflammation, leading to the creation of additional phagocytic cells (positive feedback) (19).

Direct tissue toxicity of superoxide released by the phagocytic cells remains subject of significant controversy, regardless of the many advocates of this theory. According to 
Kuyumdzhieva and Petrova, superoxide anions are minimally toxic to tissues in the presence of iron or other transition metals hydroxyl radicals may be formed causing significant tissue damage during inflammatory response (19).

A large number of authors $(25,46)$ emphasise the fact that the polymorphonuclear neutrophils and monocytes contain the enzyme myeloperoxidase (MPO), catalyzing the preparation of several strong oxidative derivatives involving $\mathrm{H}_{2} \mathrm{O}_{2}$ and halogen ions. Among MPO-derivatives in vivo, HOCL is the most common and physiological concentration of chloride $(\sim 150 \mathrm{mM})$ favours its formation $(25$; 47-49). Sugamura and John F. Keaney Jr indicated as a distinctive feature of HOCL as an oxidant the fact that it is a two-electron oxidizing agent and reacts weakly with one-electron antioxidants such as vitamin $\mathrm{E}$ (which may partially account for the failure in the use of antioxidant supplements for the prevention and treatment of atherosclerosis). (25) MPO is also involved in the transition from vulnerable plaque to plaque rupture. The mechanism includes MPO-generation of NOCL, which can lead to the activation of matrix metalloproteases and lipid peroxidation in the arterial wall $(25 ; 50-52$; 46).

Given the potential role of MPO in the atherosclerotic process, genetic polymorphisms leading to MPO deficiency or reduced activity are associated with reduced risk of cardiovascular disease, but the reason for this is unclear $(46 ; 53)$.

\section{CONCLUSION}

Despite the evidence supporting the role of oxidative stress in the pathogenesis of cardiovascular pathology in experimental animals, there is no conclusive evidence yet of its importance in human pathology. It is certain that oxidative stress is a key factor in the molecular mechanisms associated with hypertension-related cardiovascular and renal diseases, and that hypertension itself can contribute to oxidative stress. The study of the supposed role of ROS in the pathogenesis of hypertension and other cardiovascular diseases would make possible the use of a more specific therapy to reduce oxidative stress and stabilize the endothelial function.

\section{REFERENCES}

1. Gadzheva ,V., Oxidative stress, cancer and chemotherapy.monograph,2M Stara Zagora, 2007.

2. Tsvetkov, N., Bochev, G., Free-radical damage. Perspectives of antioxidant prophylaxis and therapy. Center for Inform. Medicine, 1996.

3. Harrison, D.G.,Griendling, K.K., Oxidative stress and hypertension. In:Izzo JL, Black HR, Sica DA (eds), Hypertension primer. 4 th ed., Lippincott Williams \& Wilkins, pp 201-204, 2008.

4. Cai, H., Harrison, D.G. ,Endothelial dysfunction in cardiovascular diseases: the role of oxidant stress. Circ Res., 87(10):840844, 2000.

5. Touyz, R.M. ,Reactive oxygen species and mediators of oxidative stress. In:Izzo JL, Black HR, Sica DA (eds), Hypertension primer. 4 th ed., Lippincott Williams \& Wilkins,pp 30-34, 2008.

6. Schramm, A., Matusik ,P.,Targeting NADPH oxidases in vascular pharmacology. Vascul Pharmacol., 56(5-6):216-231,2012.

7. Balasubramanyam, M., Antioxidants and Cardiovascular Disease - Where Do We Stand? The Asian Journal of diabetology,8(1):48-52, 2006.

8. Förstermann,U., Nitric oxide and oxidative stress in vascular disease. Pflugers Arch., 459(6):923-939, 2010.

9. Queisser,N., Schupp,N., Aldosterone, oxidative stress, and NF- $\mathrm{BB}$ activation in hypertension-related cardiovascular and renal diseases. Free Radic Biol Med., 53(2):314327,2012 .

10. Touyz,R.M. ,Reactive Oxygen Species, Vascular Oxidative Stress and Redox Signaling in Hypertension. What Is the Clinical Significance? Hypertension. ,44(3):248-252,2004.

11. Touyz, R.M., Tabet, F., Schiffrin, E.L., Redox-dependent signalling by angiotensin II and vascular remodelling in hypertension. Clin Exp Pharmacol Physiol.,30:860-866,2003.

12. Chiarugi,P., Cirri, P., Redox regulation of protein tyrosine phosphatases during receptor tyrosine kinase signal transduction. Trends Biochem Sci., 28: 509-551,2003.

13. Montezano,A. C.,Touyz, R. M., Reactive Oxygen Species and the Cardiovascular System. In Granger DN, Granger JP(eds), Morgan \& Claypool Publishers, January 2012.

14. Paravicini, T. M., Chrissobolis, S., Drummond, G. R., Sobey, C. G., Increased NAD(P)H-oxidase activity and Nox4 
expression during chronic hypertension is associated with enhanced cerebral vasodilatation to $\mathrm{NAD}(\mathrm{P}) \mathrm{H}$ in vivo. Stroke,35:584-589, 2004.

15. Liu, H., Li, H., Kalyanaraman, B., Nicolosi, A. C., Gutterman, D. D.,Mitochondrial sourcesof $\mathrm{H} 2 \mathrm{O} 2$ generation play a key role in flow-mediated dilation in human coronary resistance arteries. Circ Res, 93:573-580, 2003.

16. Matoba, T., Shimokawa, H., Nakashima, M., Hirakawa, Y., Mukai, Y., Hirano, K.,Hydrogen peroxide is an endotheliumderived hyperpolarizing factor in mice. J Clin Invest, 106:1521-1530, 2000.

17. Cai, H., Harrison, D. G., Endothelial dysfunction in cardiovascular diseases: the role ofoxidant stress. Circ Res, 87: 840-844, 2000.

18. Cai, H., Hydrogen peroxide regulation of endothelial function: origins, mechanisms and consequences. Cardiovasc Res, 68(1):26-36, 2005.

19. Kuyumdzhieva, A., Petrova, V.,Cellular defense mechanisms against oxidative stress, Faculty of Biology, Sofia University "St. Kliment Ohridski ", Sofia, 2010.

20. Tsutsui ,H., Kinugawa,S., Matsushima,S.,Ox idative stress and heart failure. American Journal of Physiology, 301 (6): H2181H2190, 2011.

21. Culotta, E., Koshland, D. E. Jr.,NO news is good news. Science, 258(5090):1862$1865,1992$.

22. Förstermann,U., Sessa,W.C.,Nitric oxide synthases: regulation and function. Eur Heart J. , 33(7):829-837,2012.

23. Andrew, P. J., Mayer, B.,Enzymatic function of nitric oxide synthases. Cardiovasc Res, 43: 521-531, 1999.

24. Vasquez-Vivar, J., Kalyanaraman, B., Martasek, P., Hogg, N., Masters, B. S., Karoui, H., Superoxide generation by endothelial nitric oxide synthase: the influence of cofactors. Proc Natl Acad Sci U S A, 95:9220-9225, 1998.

25. Sugamura, K., Keaney,J.F., Reactive Oxygen Species in Cardiovascular Disease. Free Radic Biol Med., 51(5): 978-992,2011.

26. Griffith ,O.W., Stuehr ,D.J. ,Nitric oxide synthases: properties and catalytic mechanism. Аnпu Rev Physiol,57:707736,1995.

27. Maia, L. B., Moura, J. J., Nitrite reduction by xanthine oxidase family enzymes: a new class of nitrite reductases. J Biol Inorg Chem ,16(3):443-460, 2011.

28. Mudau, M., Genis, A.,Endothelial dysfunction: the early predictor of atherosclerosis. Cardiovasc J Afr, 23(4):222231,2012.

29. Wilcox ,J.N., Subramanian ,R.R., Sundell, C.L., Tracey, W.R., Pollock, J.S., Harrison, D.G., Marsden ,P.A., Expression of multiple isoforms of nitric oxide synthase in normal and atherosclerotic vessels. Arterioscler Thromb Vasc Biol,17:2479-2488,1997.

30. Pou,S., Pou,W.S., Bredt ,D.S., Snyder ,S.H., Rosen,G.M. ,Generation of superoxide by purified brain nitric oxide synthase. J Biol Chem.,267:24173-24176,1992.

31. Shimokawa,H., Flavahan,N.A., Vanhoutte, P.M. ,Loss of endothelial pertussis toxinsensitive $G$ protein function in atherosclerotic porcine coronaryarteries. Circulation.,83:652660,1991.

32. Harrison, D.G. ,Endothelial function and oxidant stress. Clin Cardiol,20:11-17,1997.

33. Rubanyi ,G.M., Vanhoutte, P.M.,Superoxide anions and hyperoxia inactivate endothelium-derived relaxing factor. Am J Physiol,250:822-827,1986.

34. Thomson,L.,Trujillo,M.,Telleri,R.,Radi,R., Kinetics of cytochrome $\mathrm{c}^{2+}$ oxidation by peroxynitrite: implications for superoxide measurements in nitric oxide-producing biological systems. Arch Biochem Biophys,319:491-497,1995.

35. Mugge,A.,Elwell,J.H.,Peterson,T.E.,Hofme yer, T.G., Heistad, D.D., Harrison, D.G.,Chronic treatment with polyethyleneglycolated superoxide dismutase partially restores endothelium-dependent vascular relaxations in cholesterol-fed rabbits. Circ Res, 69:1293-1300,1991.

36. Durante,W., Sen, A.K., Sunahara ,F.A., Impairment of endothelium-dependent relaxation in aortae from spontaneously diabetic rats. $\mathrm{Br} \quad \mathrm{J}$ Pharmacol,94:463468,1988 .

37. Lockette,W., Otsuka ,Y., Carretero, O., The loss of endothelium-dependent vascular relaxation hypertension. Hypertension, 8(II):6166,1986.

38. Oyama,Y., Kawasaki,H., Hattori,Y., Kanno, M. ,Attenuation of endothelium-dependent relaxation in aorta from diabetic rats. Eur $J$ Pharmacol, 132:75-78,1986.

39. Winquist,R.J., Bunting, P.B., Baskin,E.P., Wallace, A.A.,Decreased endotheliumdependent relaxation in New Zealand genetic hypertensive rats. $J$ Hypertens, 2:541-545,1984. 
40. Sudano, I., Roas, S., Noll, G. ,Vascular abnormalities in essential hypertension. Curr Pharm Des., 17(28):3039-3044,2011.

41. Mervaala, E. M., Cheng, Z. J., Tikkanen, I., Lapatto, R., Nurminen, K., Vapaatalo, H., Endothelial dysfunction and xanthine oxidoreductase activity in rats with human renin and angiotensinogen genes. Hypertension, 37:414-418, 2001.

42. Halcox,J. P., Quyyumi, A. A. ,Endothelial function and cardiovascular disease. In :Izzo JL,Black HR,Sica DA(eds), Hypertension primer.4th ed., Lippincott Williams \& Wilkins, pp.204-208, 2008.

43. Charakida,M., O'Neil ,F.,Inflammatory disorders and atherosclerosis: new therapeutic approaches. Curr Pharm Des., 17(37):4111-4120,2011.

44. Yin.Y.,Inflammasomes: sensors of metabolic stresses for vascular inflammation. Front Biosci ,18:638649,2013.

45. Juni,R.P., Duckers,H.J.,Oxidative stress and pathological changes after coronary artery interventions. $J \quad \mathrm{Am} \quad$ Coll Cardiol.,61(14):1471-1481,2013.

46. Spagnoli,L.G.,Bonanno,E., Sangiorgi,G., Mauriello,A., Role of Inflammation in Atherosclerosis. J Nucl Med , 48:1800$1815,2007$.

47. Hazen,S.L., Heinecke, J.W., 3Chlorotyrosine, a specific marker of myeloperoxidase-catalyzed oxidation, is markedly elevated in low density lipoprotein isolated from human atherosclerotic intima. $J$ Clin Invest,99:2075-2081,1997.

48. Harrison, J.E., Schultz, J., Studies on the chlorinating activity of myeloperoxidase. $J$ Biol Chem,251:1371-1374,1976.

49. Weiss,S.J., Test,S.T.,Eckmann,C.M., Roos, D., Regiani,S., Brominating oxidants generated by human eosinophils. Science, 234:200-203,1986.

50. Daugherty, A., Dunn ,J.L., Rateri ,D.L., Heinecke, J.W., Myeloperoxidase, a catalyst for lipoprotein oxidation, is expressed in human atherosclerotic lesions. $J$ Clin Invest,94:437-444, 1994.

51. Hazell,L.J., Arnold, L., Flowers,D., Waeg, G., Malle,E., Stocker, R.,Presence of hypochlorite-modified proteins in human atherosclerotic lesions. $J$ Clin Invest, 97:1535-1544,1996.

52. Fu,X., Kassim,S.Y., Parks,W.C., Heinecke, J.W.,Hypochlorous acid oxygenates the cysteine switch domain of pro-matrilysin (MMP-7). A mechanism for matrix metalloproteinase activation and atherosclerotic plaque rupture by myeloperoxidase. J Biol Chem, 276:4127941287,2001.

53. Brennan, M.L., Penn, M.S., Van Lente, F., Prognostic value of myeloperoxidase in patients with chest pain. $N$ Engl $J$ Med,349(17):1595-1604,2003. 